\begin{tabular}{|c|c|}
\hline 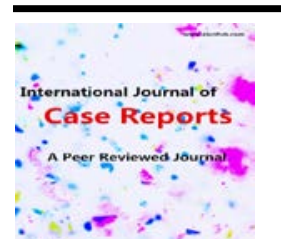 & $\begin{array}{l}\text { International Journal of Case Reports } \\
\text { (ISSN:2572-8776) }\end{array}$ \\
\hline
\end{tabular}

\title{
Primary lymphoma of bone, myeloma mimicker: a case report and
}

\section{review of literature}

\section{Deena Mudawi ${ }^{1}$, Samah Kohla ${ }^{2,3}$, Feryal Ibrahim², Hesham Elsabah ${ }^{1}$, Mohamed Abdelrazek ${ }^{4}$,} Lajos Szabados ${ }^{5}$, Mohammad A Abdulla ${ }^{1}$, Omar M Ismail ${ }^{1}$, Abdulqadir J Nashwan ${ }^{1}$ And Mohamed A Yassin ${ }^{1}$

${ }^{1}$ Department of Medical Oncology, Hematology Section, National Center for Cancer Care and Research, Doha, Qatar; ${ }^{2}$ Lab Medicine and Pathology Department, Hematology Section, Hamad Medical Corporation, Doha, Qatar; ${ }^{3}$ Clinical Pathology Department, Hematology Section, Faculty of Medicine, Al-Azhar University, Cairo, Egypt; ${ }^{4}$ Department of Radiology, Hamad Medical Corporation; ${ }^{5}$ PET/CT Center, Clinical Imaging, National Center for Cancer Care and Research, Doha, Qatar

\section{ABSTRACT}

Primary lymphoma of the bone (PLB) primarily arising from the medullary cavity is an extremely rare entity, with only limited number of studies and sporadic cases reported in the literature. The current study presents one case of PLB presented with unique presentation and treated with chemotherapy only. A 66 year old male presented with severe pain in the left hip that persisted for two months. Initial laboratory work up showed anemia, hypercalcemia and increase in free light chains kappa to lambda ratio, a diagnosis of multiple myeloma was suspected, magnetic resonance imaging of the vertebral spine extensive involvement of bone marrow of the vertebrae.

An $18 \mathrm{~F}$ fluorodeoxyglucose positron emission tomography computed tomography (FDG PET CT) scan showed widely scattered moderate to intense uptake in multiple lytic of skeleton, Impression was : Image compatible with extensive multiple myeloma, No extra osseous involvement was found.

Bone marrow examination was performed confirmed a diagnosis of extensive bone marrow involvement by High grade B-cell neoplasm with areas of bone marrow necrosis.

The patient received six cycles of $\mathrm{R}-\mathrm{CHOP}$ regimen (Rituximab, cyclo-phosphamide, Anthracycline, vincristine and prednisone) and achieved a complete response, as confirmed by FDG PET CT. At present, the patient is in a good condition.so this patient presentation mimicking multiple myeloma, which represent diagnostic dilemma.

Primary lymphoma of the bone is rare disease entity, there is no strong evidence supporting some diagnostic and therapeutic decisions, and therefore, case presentation of the rare disease may facilitate treatment of similar diseases. A review of the literature to elucidate the clinical, radiological, pathological phenotype and optimal treatment of PLB done.
Key words: Primary lymphoma of the bone, extra nodal, high grade B-cell lymphoma, multi focal lesions

${ }^{*}$ Correspondence to Author:

Dr. Mohamed A Yassin

Consultant, Hematologist

Medical Oncology/Hematology section; NCCCR HMC

How to cite this article:

Deena Mudawi, Samah Kohla, Feryal Ibrahim, Hesham Elsabah, Mohamed Abdelrazek, Lajos Szabados, Mohammad A Abdulla, Omar M Ismail, Abdulqadir J Nashwan, Mohamed A Yassin. Primary lymphoma of bone, myeloma mimicker: A case report and review of literature. International Journal of Case Reports, 2018 3:55.

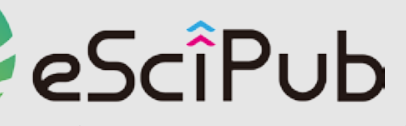

eSciPub LLC, Houston, TX USA. Website: http://escipub.com/ 


\section{Introduction}

Primary lymphoma of bone (PLB) is a rare extra nodal lymphoma that accounts for less than 1 percent of all non-Hodgkin lymphomas $(\mathrm{NHL})$ in adults, 3 to 7 percent of primary bone tumors and less than 5 percent of all extra nodal nonHodgkin lymphomas. Usually, presented as solitary or multiple and destructive bone lesions. [1-3]

The vast majority of cases of PLB are pathologically diagnosed as diffuse large $B$ cell lymphoma (DLBCL), Imaging features are usually non-specific. As PLB is a curable disease, it is important for it to be differentiated from other causes of lytic bone lesions, such as carcinomas and other primary bone tumors. [2] We report a 66-year-male presented with hip pain, investigation revealed hypercalcemia and multiple lytic bone lesions mimicking multiple myeloma, which represent diagnostic dilemma.

\section{Case report}

We report 66 -year- old male presented to emergency department with severe pain in the left hip that persisted for two months, progressive, associated with weight loss $15 \mathrm{~kg}$, but no fever or night sweats. No neurological manifestations. Physical examination revealed abed bound patient due to severe left hip pain. Bilateral hips tenderness with limited range of movement, otherwise unremarkable examination, no organomegly.

Investigations showed white blood count 12.8 $\mathrm{U} / \mathrm{L}(4-10,000 \mathrm{U} / \mathrm{L})$, hemoglobin $11.5 \mathrm{~g} / \mathrm{dl}$ (12$15 \mathrm{~g} / \mathrm{dl})$, and platelets $353 \mathrm{U} / \mathrm{L}(150-450 \mathrm{U} / \mathrm{L})$.

Peripheral smear showed moderate hypochromic microcytic anemia Mild neutrophilic leukocytosis.

Serum creatinine 57umil/L (64-110umol/L) ,calcium $2.97 \mathrm{mmol} / \mathrm{L}(2.1-2.55 \mathrm{mmol} / \mathrm{L})$, albumin $26 \mathrm{gm} / \mathrm{L} \quad(35-50 \mathrm{gm} / \mathrm{L})$, protein $72 \mathrm{gm} / \mathrm{L}(64-$ 83gm/L), LDH $438 \mathrm{U} / \mathrm{L}(125-220)$.

Beta-2 macroglobulin $3.64 \mathrm{mg} / \mathrm{L}(0.87-2.64)$, , KaFLC 99.4(3.3-19.4) LaFLC 24.9 (5.71-26), $\mathrm{Ka} / \mathrm{La}$ FLC ratio of 3.98 [0.26-1.65], Urine negative for bence-Jones Protein, serum protein electrophoresis showed no monoclonal band detected.

X-ray Pelvis and Left Hip showed diffuse osteoporosis of the bones, scattered sclerotic foci noted scattered in the visualized bones.

MRI thoracic lumbar spine showed extensive involvement of bone marrow of the vertebrae (Fig 1).

\section{FDG-PET-CT:}

Showed moderate to intense uptake in multiple lytic lesions associated with cortical breakdowns scattered widely in the imaged skeleton involving vertebrae, ribs, clavicles, scapulae, pelvic bones, and proximal 2/3 of humeri and both femora down to the knees. Impression: Image compatible with extensive multiple myeloma, No extra osseous involvement was found. Fig 2(A\&B)

Bone marrow examination performed:

Peripheral blood shows mild hypochromic microcytic anemia, $\mathrm{Hb} \quad(10.8 \mathrm{~g} / \mathrm{d} \mathrm{l})$, mild thrombocytosis (Platelets: $430 \times 10^{\wedge} 3 / \mathrm{ul}$ ) and mild leukocytosis (WBCs: $11.310^{\wedge} 3 / \mathrm{ul}$ ) with leucoerythroblastic picture. Bone marrow aspirate shows no particles and partially clotted and hemodilute with decreased shows relative increase in lymphocytes (52\%) that appear mature looking, few (4\%) looks atypical with blastoid morphology (relatively less condensed chromatin and one or more inconspicuous to prominent nucleoli). Flow cytometry on bone marrow aspirate shows a minute population of monotypic $B$ cells comprises $<1 \%$ expressing CD19, CD20, CD10, FMC7, CD11c, cBCL2, partial CD43, IgM and show Kappa light chain restriction. The cells are negative for $C D 79 b$, CD5, CD23, CD103, CD200, IgD, IgG and IgA. Touch slides is hypercellular and stuffed with many medium to large cells with very high N/C ratio, irregular nuclear borders, some show blastic morphology (less condensed chromatin and one or more inconspicuous to multiple nucleoli) (Fig 3).

Bone marrow biopsy is suboptimal with fragmentation and aspiration artifact. The 
preserved areas appear hypercellular for age with (75\% to $85 \%)$ and extensively infiltrated by medium to large sized abnormal cells, with irregular nuclear contour, some with blastic morphology with marked depression of the trilineage hematopoietic cells (Fig 4A\&B). These abnormal cells show diffuse positivity for CD20, PAX5, CD10, Bcl6, partial Bcl2 and shows $>90 \%$ positivity for Ki67 and (20\% to $30 \%)$ positivity for C-MYC (Fig 5), while negative for CD34, TdT, CD5 and CD23 with reactive increase in CD3 positive T- cells. There are areas of the biopsy show cellular necrosis with many ghost cells. There is significant increase in reticulin fibers (Grade 2/3) (Fig 6).
Patient diagnosed as Primary lymphoma of the bone and Started on R-CHOP regimen (Rituximab- Cyclophosphamide-VincristineAnthracycline-Prednisolone) chemotherapy protocol including CNS prophylaxis with intrathecal methotrexate.

Because of persistent pain and zero ambulation capability orthopedics were consulted again and $\mathrm{X}$-ray pelvis and hips were repeated, diagnosed with Pathological Left trochanteric fracture and Left femur intramedullary nailing done.

After 6 cycles of R-CHOP chemotherapy, last FDG-PET- CT showed complete metabolic remission.

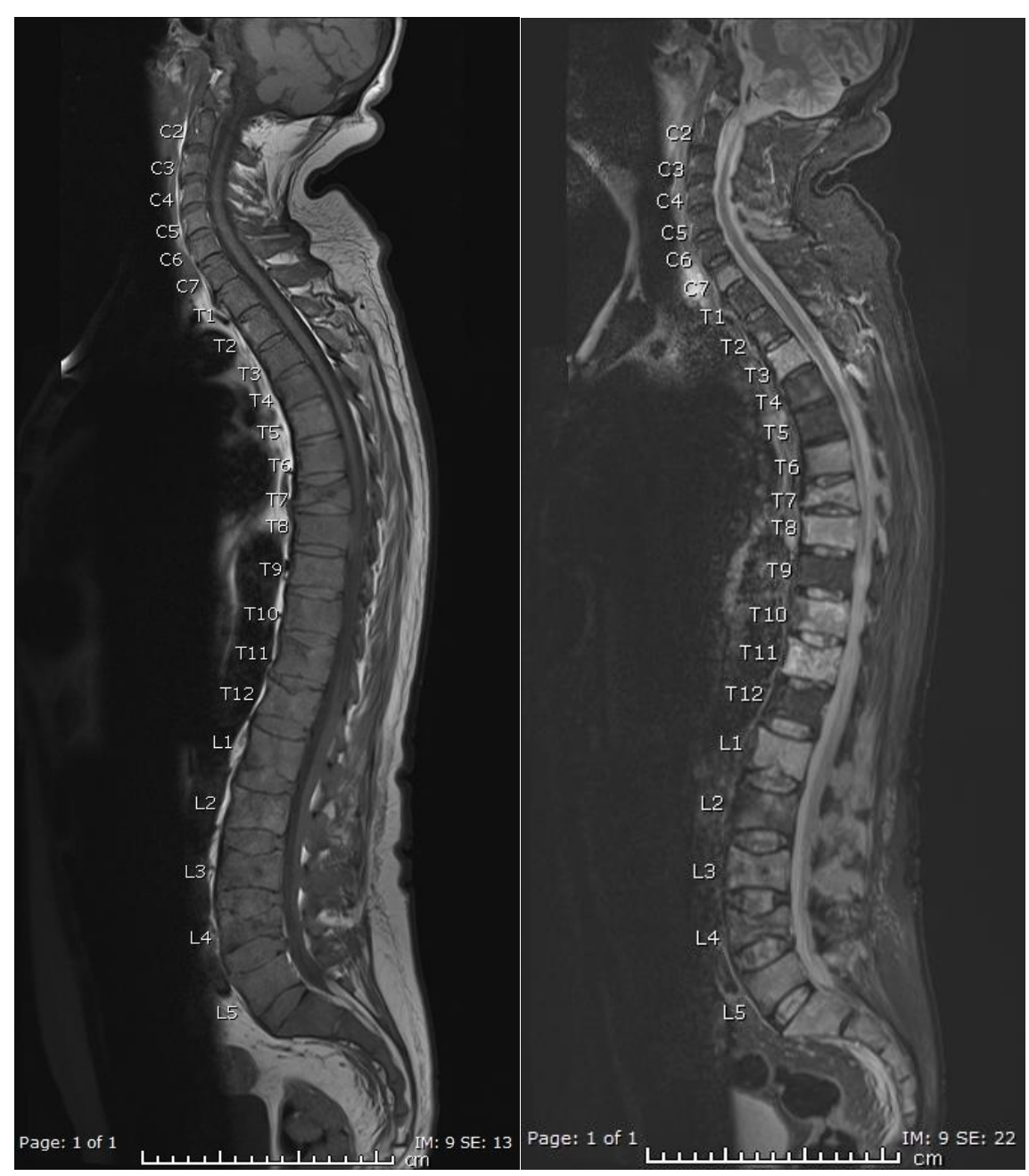

Figure 1: A: Sagittal T1 weighted images of the whole spine. B: Sagittal STIR images of the whole spine. There is extensive involvement of bone marrow of the vertebrae (involving vertebral bodies and posterior neural elements) with low signal in T1 WI and high signal in STIR images. Variable degree of vertebral collapse more marked at T7 and L4 (yellow arrows). No significant posterior displacement or neurological compression. 

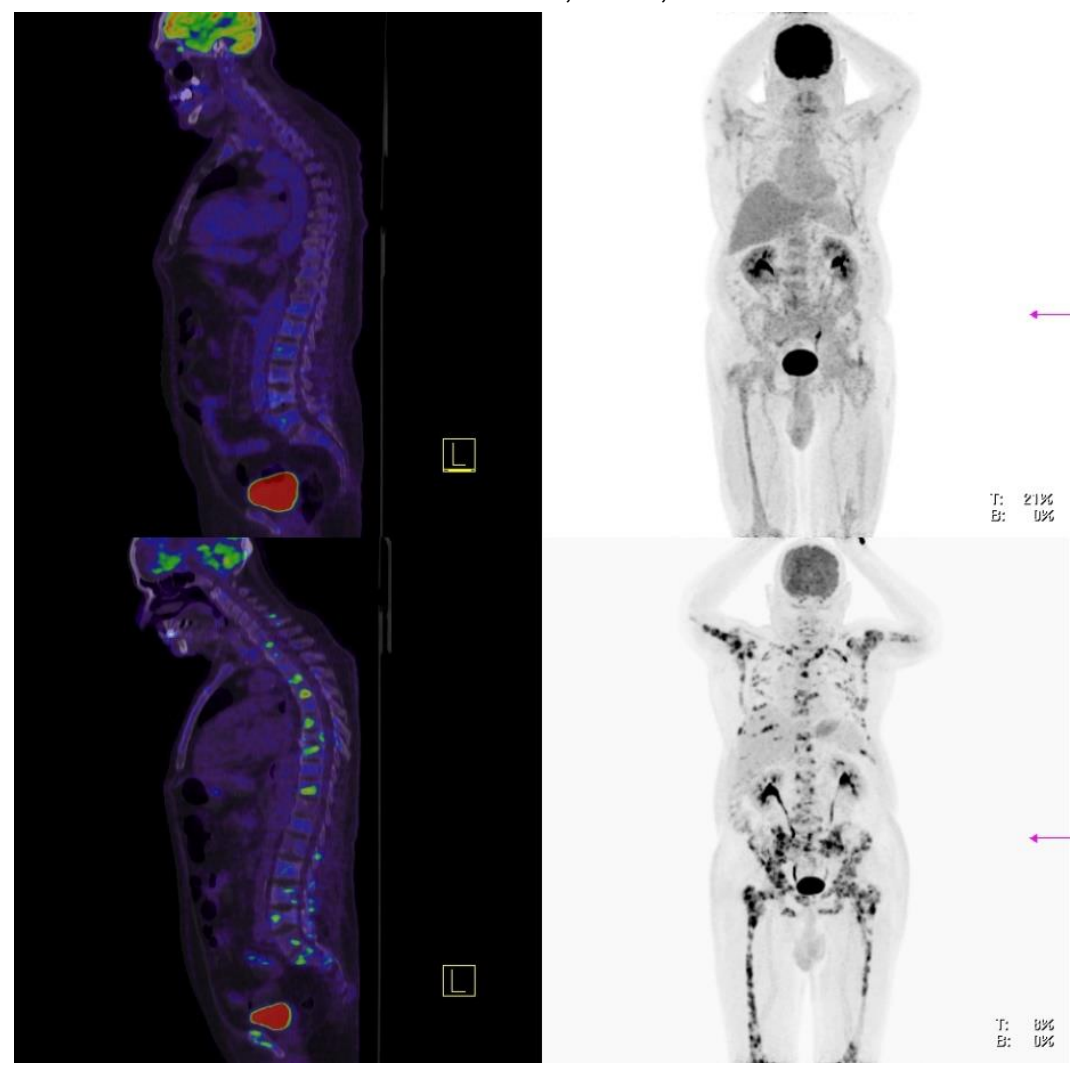

Figure 2,A

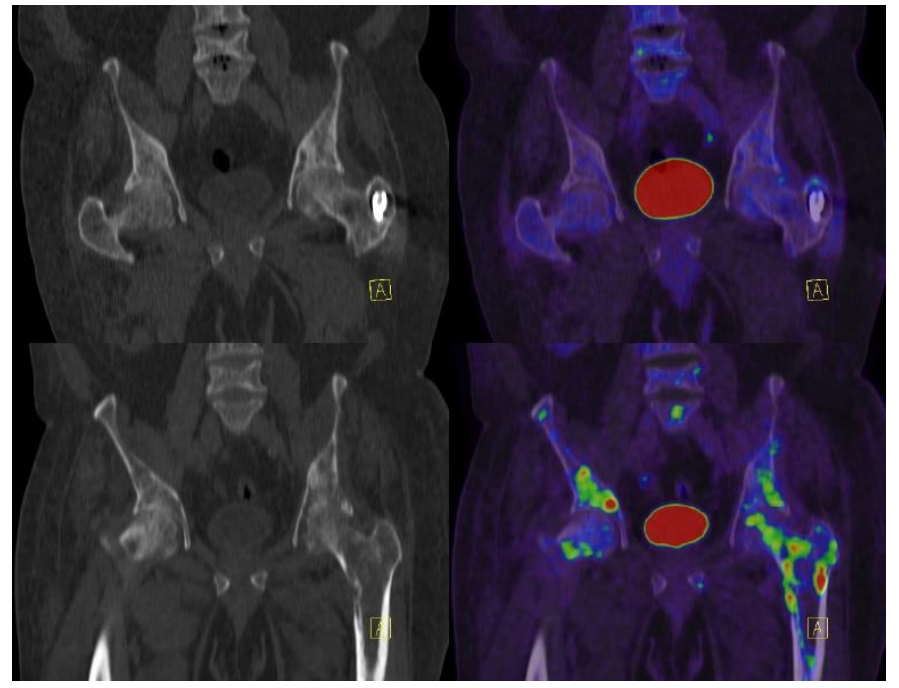

Figure 2,B

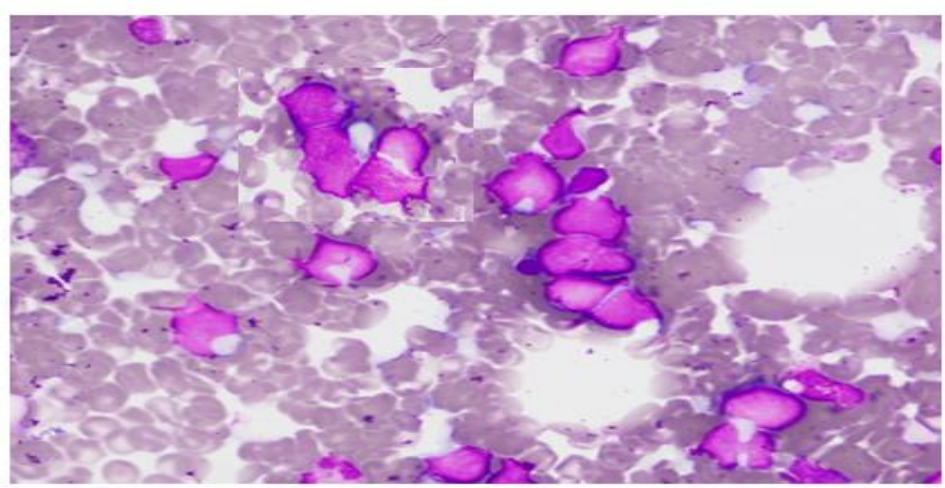

(Figure 3) x100 Touch slides is hypercellular and stuffed with many medium to large cells with very high N/C ratio, irregular nuclear borders, some show blastic morphology (less condensed chromatin and one or more inconspicuous to multiple nucleoli) 

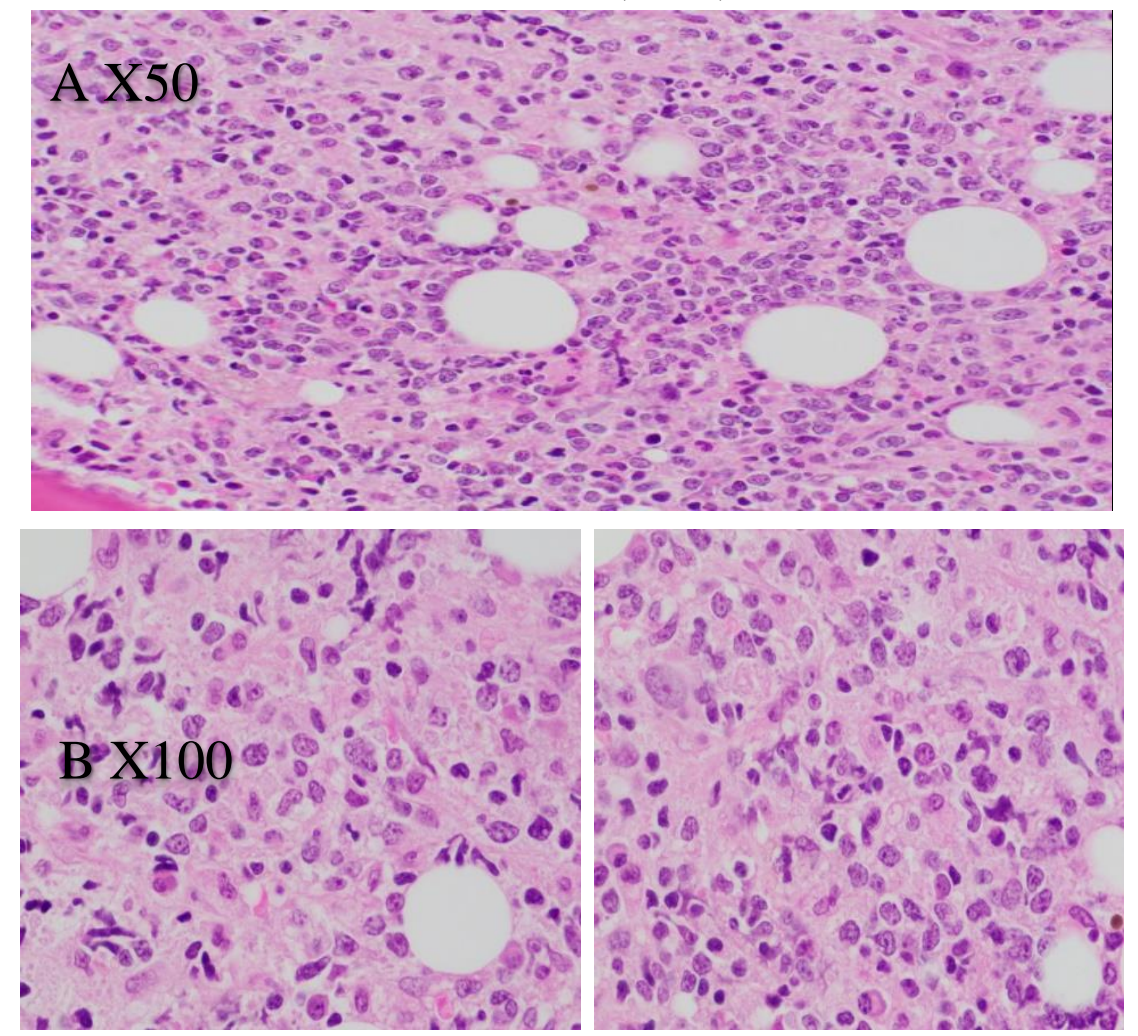

Figure 4 A \& B Bone marrow biopsy is hypercellular with (75\% to $85 \%)$ and extensively infiltrated by medium to large sized abnormal cells, with irregular nuclear contour, some with blastic morphology with marked depression of the trilineage hematopoietic cells
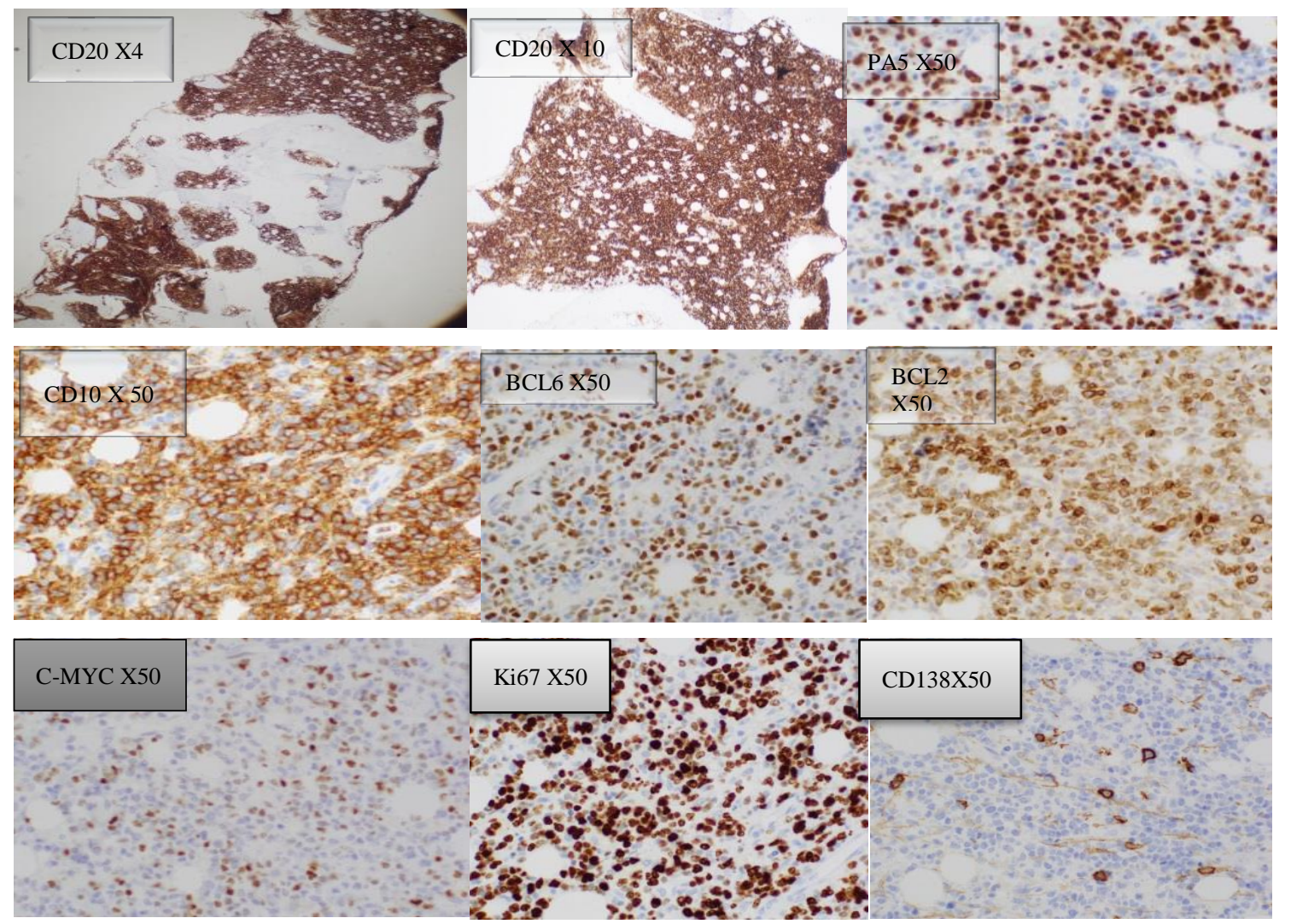

Figure 5 The abnormal cells show diffuse positivity for CD20, PAX5, CD10, Bcl6, Partial Bcl2 and shows $>90 \%$ positivity for Ki67 and (20 to $30 \%$ ) positivity for C-MYC, CD 138 X50 shows scattered plasma cells. 


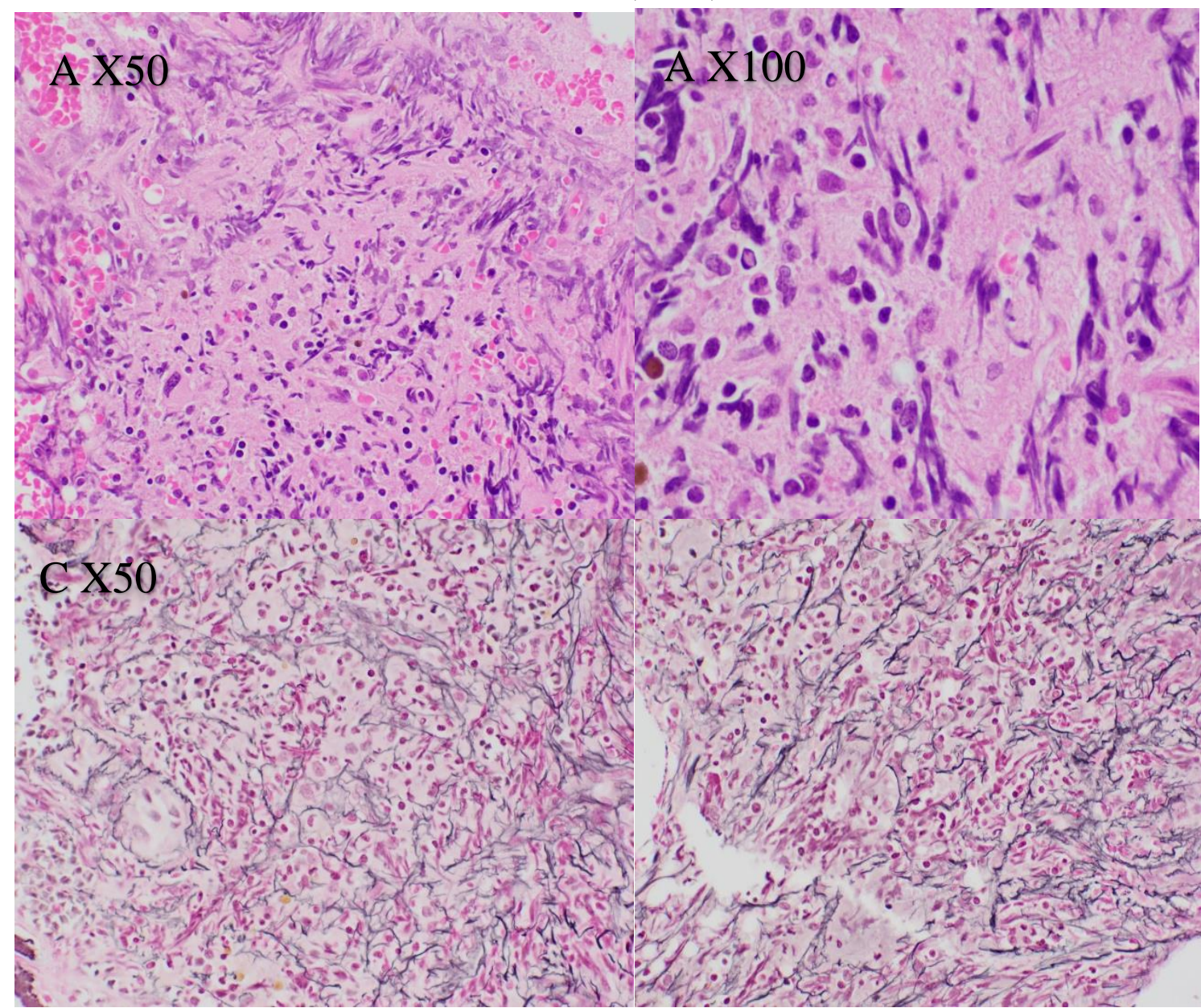

Figure 6. Increase in reticulin fibers (Grade 2/3) . H \& E (A \& B) Reticulin Stain (C)

Cytogenetic study revealed :abnormal karyotype $47, X Y,+X, t(1 ; 3)(q 42 ; q 12), \operatorname{add}(14)(q 32), \operatorname{add}(19)$ (q13.3)[7]/46,XY[43]. G-banding chromosome analysis of 50 metaphase cells from bone marrow sample revealed an abnormal clone. The abnormal clone $(7 / 50$ cells) contains an extra copy of chromosome $X$, a reciprocal translocation between chromosomes 1 and 3 at cytogenetic bands $1 \mathrm{q} 42$ and $3 \mathrm{q} 12$, additional materials of unknown origin at $14 q 32$ and 19q13. A normal clone ( $43 / 50$ cells) was also present.

\section{Discussion:}

Primary bone lymphoma (PBL) is rare accounting for approximately $4-5 \%$ of extra nodal lymphoma and less than $1 \%$ of all NonHodgkin's lymphoma. Arising from medullary cavity. The cause of PLB is not well known and any part of the skel-eton can be involved [2].

Median age at diagnosis ranges between 45 and 60 years old with a male prevalence, while pediatric cases have been also reported [8-10]. The vast majority of cases of PLB are pathologically diagnosed as diffuse large $B$ cell lymphoma (DLBCL) in 60-80 percent, rarely indolent lymphomas may present as PLB. Highly aggressive subtypes, such as Burkitt or lymphoblastic lymphoma are rare.

Patients with PLB commonly present with local bone pain, soft tissue swelling, a mass or pathological fracture, or hypercalcemic crisis. 'B' symptoms are rare and are only observed in stage IV patients. Staging varies with different diagnosing criteria at different times [2].

Diagnosis of PBL depends on the combined clinical examination and imaging studies and is confirmed with immunohistochemically examination.

Radiologically bony lesions may be extensive, destructive or infiltrative [1]. The lytic destructive pattern is the most common radiographic appearance of PBL and it was reported in 
approximately $70 \%$ of 237 cases reviewed by Mulligan et al [3]. Cortical breakthrough, pathological fracture and soft tissue masses represent a more aggressive pattern of involvement and poor prognosis [1]

Owing to its rarity, only a few retrospective studies have been published addressing the prognosis and treatment of primary bone lymphoma .As primary bone lymphoma is a highly curable disease, differentiation from other causes of lytic bone lesion such as secondaries from carcinomas and other primary bone tumors is important. Among PLBs, DLBCL is the most common subtype and accounts for $6880 \%$ of the cases. The current standard chemotherapy for patients with DLBCL consists of cyclophosphamide, doxorubicin, vincristine and prednisone $(\mathrm{CHOP})$ or $\mathrm{CHOP}$ with rituximab $(\mathrm{R}$ CHOP). [11-13] Over the last two decades, chemotherapy (CHOP based) has become the standard treatment for PBL with or without consolidative irradiation. Traditionally, irradiation has been used alone or in combination with chemotherapy. [11-13] Irradiation alone in limited-stage disease has resulted in a poor fiveyear overall survival (OS) of around $45 \%$ even when patients have been carefully chosen for apparently limited-stage disease [9]

As regards the treatment outcome of PLB, combination chemotherapy with local radiotherapy has been reported to be superior to radiotherapy alone. Role of radiation in advanced stage PBL is controversial. [9]

Conversely, surgical resection, as a local treatment, has a limited indication only for spinal cases with progressive neurological disorder or cases with pathological fractures in the extremities .In the present case, chemotherapy with $\mathrm{R} C H O P$ was given and the tumor exhibited a CR; therefore, these treatments were considered to be beneficial. Our case presented with hip pain and, on evaluation, the patient was diagnosed with primary bone lymphoma (DLBCL). The features included: serum hypercalcemia, multifocal osteolytic lesions present all over the body, and $\mathrm{NHL}$ presenting as a primary bone tumor with multifocal disease is extremely rare. Primary bone lymphoma should be considered in the differential diagnosis of multiple osteolytic bony lesions.

\section{Conclusion}

In conclusion, PLB is exceedingly rare and its radiological findings are variable and non specific; therefore, diagnosis of $\mathrm{PBL}$ depends on the combined clinical examination, imaging studies, and confirmed with immunohistochemically examination.

Since PLB in the early stages is relatively curable by appropriate multimodal treatment using chemo and radiotherapy, correct diagnosis and staging by histological and imaging examinations are crucial. When radiologically diagnosing bone tumors clinicians should consider the possibility of PBL.

\section{References}

1. Rahmat K, Wastie ML, Abdullah BJ. Primary bone lymphoma: report of a case with multifocal skeletal involvement. Biomedical imaging and intervention journal. 2007 Oct;3(4).

2. Zhou HY, Gao F, Bu B, Fu Z, Sun XJ, Huang CS, Zhou DG, Zhang $S$, Xiao J. Primary bone lymphoma: A case report and review of the literature. Oncology letters. 2014 Oct 1;8(4):15516.

3. Mulligan ME, McRae GA, Murphey MD. Imaging features of primary lymphoma of bone. AJR. American journal of roentgenology. 1999 Dec;173(6):1691-7.

4. Elkourashy SA, Nashwan AJ, Alam SI, Ammar AA, Sayed AM, Omri HE, Yassin MA. Aggressive lymphoma "Sarcoma Mimicker" originating in the gluteus and adductor muscles: a case report and literature review. Clinical Medicine Insights: Case Reports. 2016 Sep;9:CCRep-S39052.

5. Wang J, Fan S, Liu J, Song B. A rare case report of primary bone lymphoma and a brief review of the literature. OncoTargets and therapy. 2016;9:4923.

6. Mandal PK, Baul S, Dolai TK. Primary bone lymphoma with multifocal osteolytic lesions: a rare case report with review of literature. Blood research. 2015 Dec 1;50(4):256-60.

7. Economopoulos $T$, Papageorgiou S, Rontogianni D, Kaloutsi V, Fountzilas G, Tsatalas C, Pavlidis N, Pectasides D, Papageorgiou E, Dimopoulos M. Multifocal extranodal non-hodgkin lymphoma: a clinicopathologic study of 37 cases in Greece, a 
Hellenic Cooperative Oncology Group study. The Oncologist. 2005 Oct 1;10(9):734-8.

8. Kaiafa $G$, Didangelos $T$, Bobos M, Karlafti $E$, Ztriva E, et al. (2018) Primary Bone Diffuse Large B-cell Lymphoma with Multifocal Osteolytic Lesions: A Rare Entity. Gen Med (Los Angeles) 6: 308. DOI: $10.4172 / 2327-5146.1000308$

9. Singh $T$, Satheesh $C$, Lakshmaiah $K$, Suresh $T$, Babu G, Lokanatha D, Jacob L, Halkud R. Primary bone lymphoma: a report of two cases and review of the literature. Journal of Cancer Research and Therapeutics. 2010 Jul 1;6(3):296-.

10. Gregory T, Theodoros G. $P$, Ismini M, Vasilios $T$, Aliki F. A Rare Case of a Non-Hodgkin's Lymphoma Presented as a Single Bone Lesion. Biomed J Sci \&Tech Res 3(2)- 2018. BJSTR.MS.ID.000861. DOI: 10.26717/BJSTR.2018.03.000861

11. Sato $S$, Kaneuchi $Y$, Hakozaki M, Yamada $H$, Kawana S, Hasegawa O, Konno S. Primary malignant lymphoma of the talus: A case report and review of the literature. Molecular and clinical oncology. 2017 Oct 1;7(4):574-8.

12. Heyning $\mathrm{FH}$, Hogendoorn PC, Kramer $\mathrm{MH}$, Hermans J, Kluin-Nelemans JC, Noordijk EM, Kluin PM. Primary non-Hodgkin's lymphoma of bone: a clinicopathological investigation of 60 cases. Leukemia. 1999 Dec;13(12):2094.

13. Ramadan KM, Shenkier T, Sehn LH, Gascoyne RD, Connors JM. A clinicopathological retrospective study of 131 patients with primary bone lymphoma: a population-based study of successively treated cohorts from the British Columbia Cancer Agency. Annals of Oncology. 2006 Oct 3;18(1):129-35. 